\title{
Far-Field Radar Cross-Section Determination from Near-Field 3-D Synthetic Aperture Imaging with Arbitrary Antenna Scanning Surfaces
}

This paper was downloaded from TechRxiv (https://www.techrxiv.org).

\section{LICENSE}

CC BY 4.0

SUBMISSION DATE / POSTED DATE

$13-10-2021 / 05-01-2022$

\section{CITATION}

Watanabe, Takuma; Yamada, Hiroyoshi (2021): Far-Field Radar Cross-Section Determination from Near-Field 3-D Synthetic Aperture Imaging with Arbitrary Antenna Scanning Surfaces. TechRxiv. Preprint. https://doi.org/10.36227/techrxiv.16802419.v2

DOI 


\title{
Far-Field Radar Cross-Section Determination from Near-Field 3-D Synthetic Aperture Imaging with Arbitrary Antenna Scanning Surfaces
}

\author{
Takuma Watanabe and Hiroyoshi Yamada, Member, IEEE
}

\begin{abstract}
In this study, we propose a generalized algorithm for far-field radar cross-section determination by using 3-D synthetic aperture imaging with arbitrary antenna scanning surfaces. This method belongs to a class of techniques called image-based near-field-to-far-field transformation. The previous image-based approaches have been formulated based on a specific antennascanning trajectory or surface, such as a line, plane, circle, cylinder, and sphere; majority of these approaches consider 2$D$ radar images to determine the azimuth radar cross-section. We generalize the conventional image-based technique to accommodate an arbitrary antenna-scanning surface and consider a 3-D radar image for radar cross-section prediction in both the azimuth and zenith directions. We validate the proposed algorithm by performing numerical simulations and anechoic chamber measurements.
\end{abstract}

Index Terms-Near-field-to-far-field transformation, radar cross-section, synthetic aperture radar, image reconstruction

\section{INTRODUCTION}

$\mathbf{R}$ ADAR cross-section (RCS) is an essential quantity in characterizing a target or an object to be measured using radar systems. Because RCS is defined in the far-field distance, which is proportional to the electrical size of the target under test, direct RCS measurements of an electrically large target require a considerably long distance between the target and the radar antenna. However, the spatial requirement and the cost of the measurement facility for the direct RCS measurement of an electrically large target are often unacceptable, and an alternative method must be developed to measure or predict the RCS in a shorter antenna distance.

To achieve this, various signal processing algorithms have been proposed to convert the scattered wave measured in the near-field into the far-field data, and these techniques are known as the near-field-to-far-field transformation (NFFFT). These well-known approaches are as follows: the physicaloptics-approximation-based method [1], plane-wave synthesis [2]-[4], and image-based method [5]-[15]. The image-based method is used in this research. As mentioned in [9]-[13], one of the challenges in the image-based method is the measurement of a strongly asymmetric target under a shorter

Manuscript received $\mathrm{xxxx} \mathrm{xx}, \mathrm{xxxx}$; revised $\mathrm{xxxx} \mathrm{xx}, \mathrm{xxxx}$; accepted $\mathrm{xxxx}$ $\mathrm{xx}, \mathrm{xxxx}$. Date of publication $\mathrm{xxxx} \mathrm{xx}, \mathrm{xxxx}$; date of current version $\mathrm{xxxx}$ $\mathrm{xx}, \mathrm{xxxx}$. The Associate Editor coordinating the review process was X. Xxxx (Corresponding author: Takuma Watanabe.)

T. Watanabe is currently with Fujitsu System Integration Laboratories Ltd., Kawasaki 2118588, Japan (e-mail: takuma.watanabe.jp@ieee.org).

H. Yamada is with the Smart Information Systems Program, Faculty of Engineering, Niigata University, Niigata 9502181, Japan.

Digital Object Identifier xxxxx/xxxxx antenna separation from the target. The accuracy of RCS prediction is degraded if no correction is taken into account for this condition in the imaging process. In [9], the correction factor was introduced in 2-D image reconstruction with a circular scanning trajectory around the target such that the integral transformation in radar imaging is self-consistent for every electrically small scatterer. In [10]-[13], this correction factor was extended to cylindrical scanning geometries, where [12] and [13] considered a 3-D imaging problem to obtain the RCS in the zenith or elevation direction.

In this study, we propose a generalization of the aforementioned image-based NFFFT algorithms with a correction factor. We consider an arbitrary antenna scanning surface for the near-field measurement, including a canonical surface, such as a plane, cylinder, and sphere, and any other numerically described surfaces for which an analytical expression may be unavailable. We derive a general expression for the correction factor that can be used for an arbitrary scanning surface and the specific correction factors for the planar, cylindrical, and spherical scanning surfaces as special cases. Furthermore, we formulate a numerical computation scheme for the correction factor, and it enables the use of a numerically described scanning surface. The derivation of this generalized correction factor is a major novelty and contribution of this study.

As we consider 2-D scanning surfaces in the 3-D spatial domain, the 3-D radar image is utilized instead of the 2D image to derive the far-field RCS. Image-based methods using circular scanning assume that the height of the target is sufficiently small such that the far-field criterion is satisfied with respect to the height dimension. By generating a 3D radar image with a 2-D scanning geometry, such as a cylindrical or spherical surface, we can remove this restriction [10]-[13]. Moreover, the NFFFT based on the 3-D image can produce the RCS pattern in both the azimuth and zenith (or elevation) directions [12], [13], and the location of the scattering source can be determined in 3-D spatial domain.

The remainder of this paper is organized as follows. In Section II, we present the formulation of the proposed imagebased NFFFT algorithm. Section III details the numerical simulation of the proposed NFFFT algorithm, using a simple model consisting of point scatterers. A spherical scanning surface is used for the demonstration. Section IV presents the experimental validation performed in an anechoic chamber. In this experiment, we apply cylindrical antenna scanning and employ a metallic aircraft model as the test target. Finally, Section V summarizes the study presented in this paper. 


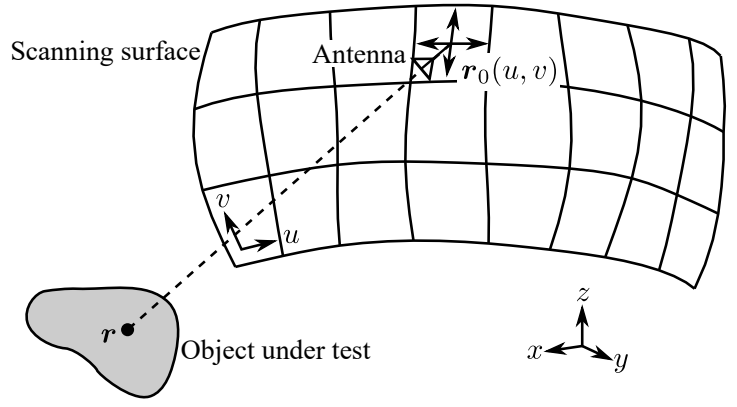

Fig. 1. System model of the synthetic aperture imaging with an arbitrary antenna scanning surface.

\section{Problem Formulation}

In this section, we discuss the formulation of the proposed NFFFT algorithm. We considered a monostatic scattering experiment in which the transmitting and receiving antennas were scanned over an arbitrary surface around the target under investigation. For this measurement configuration, we derived a correction factor used in the image reconstruction procedure, which improved the accuracy of the following RCS computation.

\section{A. System and Signal Model}

Fig. 1 depicts the system model of the synthetic aperture imaging with an arbitrary antenna-scanning surface. We consider a measurement in the 3-D spatial domain $(x, y, z)$, and denote a general location in this space as $r$. The transmitting and the receiving antennas are located on an arbitrary scanning surface $\boldsymbol{r}_{0}(u, v)$ parametrized by the variables $(u, v)$. These vectors $\boldsymbol{r}$ and $\boldsymbol{r}_{0}(u, v)$ are defined as follows:

$$
\begin{aligned}
\boldsymbol{r} & =x \widehat{\boldsymbol{x}}+y \widehat{\boldsymbol{y}}+z \widehat{\boldsymbol{z}} \\
\boldsymbol{r}_{0}(u, v) & =x_{0}(u, v) \widehat{\boldsymbol{x}}+y_{0}(u, v) \widehat{\boldsymbol{y}}+z_{0}(u, v) \widehat{\boldsymbol{z}},
\end{aligned}
$$

where $\widehat{\boldsymbol{x}}, \widehat{\boldsymbol{y}}$, and $\widehat{\boldsymbol{z}}$ are the unit vectors along the $x-, y$-, and $z$-directions, respectively. For simplicity, we assume a simplyconnected closed surface for $\boldsymbol{r}_{0}(u, v)$, which completely encloses the spatial domain to be imaged. However, an open surface and a discrete set of antenna locations can be employed for the measurement and image reconstruction, as discussed in Section II-C and Section II-E.

The transmitting antenna at location $\boldsymbol{r}_{0}(u, v)$ on the scanning surface emits an electromagnetic wave, and the receiving antenna at the same location collects the scattered wave. This measurement procedure was repeated for all desired antenna locations. We define the angular frequency of the transmitted wave as $\omega$ and the corresponding wavenumber as $k=\omega / c$, where $c$ is the wave-propagation speed.

If we denote the reflection coefficient at location $\boldsymbol{r}$ by $C(\boldsymbol{r})$, which is distributed over the spatial volume $V$, the received signal can be expressed as

$$
E^{s}\left(k, \boldsymbol{r}_{0}\right)=\frac{k^{2}}{\sqrt{4 \pi}} \int_{V} P^{2}\left(k, \boldsymbol{r}_{0}, \boldsymbol{r}\right) C(\boldsymbol{r}) \frac{e^{-2 j k\left|\boldsymbol{r}_{0}-\boldsymbol{r}\right|}}{\left|\boldsymbol{r}_{0}-\boldsymbol{r}\right|^{2}} d \boldsymbol{r},
$$

where $P\left(k, \boldsymbol{r}_{0}, \boldsymbol{r}\right)$ is the directive pattern of the antenna in the direction from $\boldsymbol{r}_{0}$ to $\boldsymbol{r}$. If the target is a cloud of discrete point scatterers located at $\boldsymbol{r}_{i}, i \in\{1,2, \ldots\}$, the reflection coefficient $C(\boldsymbol{r})$ can be defined as

$$
C(\boldsymbol{r})=\sum_{i} C_{i} \delta\left(\left|\boldsymbol{r}-\boldsymbol{r}_{i}\right|\right)
$$

where $C_{i}$ represents the reflectivity of the $i$ th point scatterer and $\delta(\cdot)$ is the delta function. For electrically small spheres of radii $a_{i}\left(k a_{i}<0.4\right)$, we can use the following expression for $C_{i}$ [9], [16]:

$$
C_{i}=3 \sqrt{\pi} a_{i}^{3}
$$

Substituting Eq. (3) into Eq. (2) yields the following received signal model for the discrete scatterers.

$$
E^{s}\left(k, \boldsymbol{r}_{0}\right)=\frac{k^{2}}{\sqrt{4 \pi}} \sum_{i} P^{2}\left(k, \boldsymbol{r}_{0}, \boldsymbol{r}_{i}\right) C_{i} \frac{e^{-2 j k\left|\boldsymbol{r}_{0}-\boldsymbol{r}_{i}\right|}}{\left|\boldsymbol{r}_{0}-\boldsymbol{r}_{i}\right|^{2}} .
$$

The use of the discrete scatterer model in Eq. (5) to test the proposed algorithm is described in Section III.

\section{B. Image Reconstruction}

The first step of image-based NFFFT is to reconstruct a spatial image from the received signal. We considered an ideal 3-D spatial image $\psi(\boldsymbol{r})$ of an electrically small scatterer (a point scatterer) at location $\boldsymbol{r}_{1}=\left(x_{1}, y_{1}, z_{1}\right)$. According to Eq. (3) with $i \in\{1\}$, it is defined as

$$
\psi(\boldsymbol{r})=C_{1} \delta\left(x-x_{1}\right) \delta\left(y-y_{1}\right) \delta\left(z-z_{1}\right) .
$$

Our objective was to reconstruct the 3-D spatial image $\psi(\boldsymbol{r})$ of Eq. (6) from the measured scattered signal $E^{s}\left(k, \boldsymbol{r}_{0}\right)$ of Eq. (5) with $i \in\{1\}$. The general expression for the image reconstruction is given by the following integral transformation of the received signal $E^{s}\left(k, \boldsymbol{r}_{0}\right)$ :

$$
\psi(\boldsymbol{r})=\int_{0}^{\infty} \int_{D_{v}} \int_{D_{u}} E^{s}\left(k, \boldsymbol{r}_{0}\right) F\left(k, \boldsymbol{r}_{0}, \boldsymbol{r}\right) d u d v d k,
$$

where $D_{v}$ and $D_{u}$ represent the domains of the integration of $u$ and $v$, respectively. The weighting function $F\left(k, \boldsymbol{r}_{0}, \boldsymbol{r}\right)$ is referred to as the focusing factor and is given by

$$
F\left(k, \boldsymbol{r}_{0}, \boldsymbol{r}\right)=g\left(\boldsymbol{r}_{0}, \boldsymbol{r}\right) \frac{\left|\boldsymbol{r}_{0}-\boldsymbol{r}\right|^{2}}{P^{2}\left(k, \boldsymbol{r}_{0}, \boldsymbol{r}\right)} e^{2 j k\left|\boldsymbol{r}_{0}-\boldsymbol{r}\right|} .
$$

In Eq. (8), $g\left(\boldsymbol{r}_{0}, \boldsymbol{r}\right)$ is the correction factor to be determined. Note that the conventional standard formulation of the synthetic aperture imaging corresponds to $g\left(\boldsymbol{r}_{0}, \boldsymbol{r}\right)=1$.

\section{Correction Factor}

Next, we derive the correction factor $g\left(\boldsymbol{r}_{0}, \boldsymbol{r}\right)$ in Eq. (8). The derivation is similar to that discussed in [9], [12], [13], except that the problem is generalized to 3-D imaging with an arbitrary scanning surface. The correction factor should be appropriately selected so that the integral transformation of Eq. (7) is self-consistent for every electrically small scatterer; that is, Eq. (7) should be reduced to the Eq. (6) corresponding to an ideal image.

According to Eq. (5) with $i \in\{1\}$, we consider the scattered wave produced by a point scatterer located at $\boldsymbol{r}_{1}$ as

$$
E^{s}\left(k, \boldsymbol{r}_{0}\right)=P^{2}\left(k, \boldsymbol{r}_{0}, \boldsymbol{r}_{1}\right) \frac{k^{2} C_{1}}{\sqrt{4 \pi}} \frac{e^{-2 j k\left|\boldsymbol{r}_{0}-\boldsymbol{r}_{1}\right|}}{\left|\boldsymbol{r}_{0}-\boldsymbol{r}_{1}\right|^{2}} .
$$




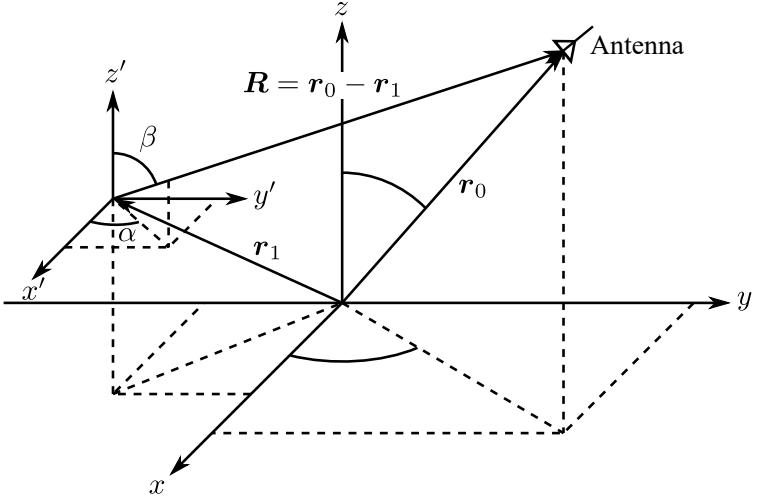

Fig. 2. Local coordinate system centered at the scatter location $\boldsymbol{r}_{1}$.

Substituting the received signal of Eq. (9) into Eq. (7) yields the following equation:

$$
\begin{aligned}
\psi(\boldsymbol{r})= & \frac{C_{1}}{\sqrt{4 \pi}} \int_{0}^{\infty} \int_{D_{v}} \int_{D_{u}} g\left(\boldsymbol{r}_{0}, \boldsymbol{r}\right) \frac{P^{2}\left(k, \boldsymbol{r}_{0}, \boldsymbol{r}_{1}\right)}{P^{2}\left(k, \boldsymbol{r}_{0}, \boldsymbol{r}\right)} \\
& \cdot \frac{\left|\boldsymbol{r}_{0}-\boldsymbol{r}\right|^{2}}{\left|\boldsymbol{r}_{0}-\boldsymbol{r}_{1}\right|^{2}} e^{j 2 k s} k^{2} d u d v d k \\
s= & \left|\boldsymbol{r}_{0}-\boldsymbol{r}\right|-\left|\boldsymbol{r}_{0}-\boldsymbol{r}_{1}\right| .
\end{aligned}
$$

Here, we consider the local coordinate system $\left(x^{\prime}, y^{\prime}, z^{\prime}\right)$, the basis vectors for which are same as those for the global coordinate system $(x, y, z)$, centered at the scatterer location $\boldsymbol{r}_{1}$ as shown in Fig. 2. We define vector $\boldsymbol{R}$ from point $\boldsymbol{r}_{1}$ to the antenna at $\boldsymbol{r}_{0}(u, v)$ as

$$
\boldsymbol{R}(u, v)=\boldsymbol{r}_{0}(u, v)-\boldsymbol{r}_{1}=R_{x} \widehat{\boldsymbol{x}}+R_{y} \widehat{\boldsymbol{y}}+R_{z} \widehat{\boldsymbol{z}},
$$

and the distance between the scatterer at $\boldsymbol{r}_{1}$ and the antenna at $\boldsymbol{r}_{0}(u, v)$ as

$$
R(u, v)=|\boldsymbol{R}(u, v)|=\sqrt{R_{x}^{2}+R_{y}^{2}+R_{z}^{2}} .
$$

In this local coordinate system, the azimuth angle $\alpha(u, v)$ and zenith angle $\beta(u, v)$ of the antenna are defined as

$$
\begin{aligned}
\alpha(u, v) & =\tan ^{-1}\left[R_{y}(u, v) / R_{x}(u, v)\right] \\
\beta(u, v) & =\tan ^{-1}\left[\rho(u, v) / R_{z}(u, v)\right] \\
\rho(u, v) & =\sqrt{R_{x}^{2}(u, v)+R_{y}^{2}(u, v) .}
\end{aligned}
$$

Note that, throughout this study, the arctangent $\tan ^{-1}(\cdot / \cdot)$ is assumed to be calculated within the range $(-\pi, \pi]$, depending on the signs of the numerator and denominator.

Using the angles $\alpha$ and $\beta$, we can approximate the function $s$ in the vicinity of the scatterer at $\boldsymbol{r}_{1}$ as [9], [12], [13]

$s \approx\left(x-x_{1}\right) \sin \beta \cos \alpha+\left(y-y_{1}\right) \sin \beta \sin \alpha+\left(z-z_{1}\right) \cos \beta$.

Substituting Eq. (14) into Eq. (10) yields the following approximation of the spatial image:

$$
\begin{aligned}
\psi(\boldsymbol{r}) \approx & \frac{C_{1}}{\sqrt{4 \pi}} \int_{0}^{\infty} \int_{D_{v}} \int_{D_{u}} g\left(\boldsymbol{r}_{0}, \boldsymbol{r}\right) \\
& \cdot \exp \left[j 2 k\left(x-x_{1}\right) \sin \beta \cos \alpha\right] \\
& \cdot \exp \left[j 2 k\left(y-y_{1}\right) \sin \beta \sin \alpha\right]
\end{aligned}
$$

$$
\cdot \exp \left[j 2 k\left(z-z_{1}\right) \cos \beta\right] k^{2} d u d v d k .
$$

In Eq. (15), we perform the integration by substitution using the following spatial frequency with respect to the local coordinate centered at point $\boldsymbol{r}_{1}$ :

$$
k_{x}=2 k \sin \beta \cos \alpha, k_{y}=2 k \sin \beta \sin \alpha, k_{z}=2 k \cos \beta .
$$

If the scanning surface is a closed surface that completely encloses the area to be imaged, the ranges of the local azimuth angle $\alpha$ and zenith angle $\beta$ are $[0,2 \pi]$ and $[0, \pi]$, respectively. Therefore, the range of integration with respect to spatial frequency variables $k_{x}, k_{y}$, and $k_{z}$ is $[-\infty, \infty]$. A similar reasoning can be applied to an open surface, that is, the surface can be extended virtually to make it a closed surface and the received signal at the extended part of the surface can be considered zero.

Using Eq. (16) in Eq. (15), we obtain the following equation:

$$
\begin{aligned}
\psi(\boldsymbol{r})= & \frac{C_{1}}{\sqrt{4 \pi}} \int_{-\infty}^{\infty} \int_{-\infty}^{\infty} \int_{-\infty}^{\infty} g\left(\boldsymbol{r}_{0}, \boldsymbol{r}\right) \\
& \cdot e^{j k_{x}\left(x-x_{1}\right)+j k_{y}\left(y-y_{1}\right)+j k_{z}\left(z-z_{1}\right)} k^{2} /|J| d k_{x} d k_{y} d k_{z},
\end{aligned}
$$

where $J$ represents the Jacobian and is given by

$$
\begin{aligned}
J & =\frac{\partial\left(k_{x}, k_{y}, k_{z}\right)}{\partial(k, u, v)}=\left|\begin{array}{lll}
\frac{\partial k_{x}}{\partial k} & \frac{\partial k_{x}}{\partial u} & \frac{\partial k_{x}}{\partial v} \\
\frac{\partial k_{y}}{\partial k} & \frac{\partial k_{y}}{\partial u} & \frac{\partial k_{y}}{\partial v} \\
\frac{\partial k_{z}}{\partial k} & \frac{\partial k_{z}}{\partial u} & \frac{\partial k_{z}}{\partial v}
\end{array}\right| \\
& =-8 k^{2} \sin \beta\left(\alpha_{u} \beta_{v}-\alpha_{v} \beta_{u}\right) .
\end{aligned}
$$

In Eq. (18), we have used the following definitions:

$$
\begin{aligned}
\alpha_{u} & =\frac{\partial \alpha}{\partial u}=\frac{1}{\rho^{2}}\left(R_{x} y_{0 u}-R_{y} x_{0 u}\right) \\
\alpha_{v} & =\frac{\partial \alpha}{\partial v}=\frac{1}{\rho^{2}}\left(R_{x} y_{0 v}-R_{y} x_{0 v}\right) \\
\beta_{u} & =\frac{\partial \beta}{\partial u}=\frac{1}{R^{2}}\left(R_{z} \rho_{u}-\rho z_{0 u}\right) \\
\beta_{v} & =\frac{\partial \beta}{\partial v}=\frac{1}{R^{2}}\left(R_{z} \rho_{v}-\rho z_{0 v}\right) \\
\rho_{u} & =\frac{\partial \rho}{\partial u}=\frac{1}{\rho}\left(R_{x} x_{0 u}+R_{y} y_{0 u}\right) \\
\rho_{v} & =\frac{\partial \rho}{\partial v}=\frac{1}{\rho}\left(R_{x} x_{0 v}+R_{y} y_{0 v}\right) \\
\chi_{\xi} & =\frac{\partial \chi(u, v)}{\partial \xi}, \chi \in\left\{x_{0}, y_{0}, z_{0}\right\}, \quad \xi \in\{u, v\} .
\end{aligned}
$$

By comparing the integral form of the 3-D delta function,

$$
\begin{gathered}
\delta\left(x-x_{1}\right) \delta\left(y-y_{1}\right) \delta\left(z-z_{1}\right)=\frac{1}{(2 \pi)^{3}} \int_{-\infty}^{\infty} \int_{-\infty}^{\infty} \int_{-\infty}^{\infty} \\
\cdot e^{j k_{x}\left(x-x_{1}\right)+j k_{y}\left(y-y_{1}\right)+j k_{z}\left(z-z_{1}\right)} d k_{x} d k_{y} d k_{z},
\end{gathered}
$$

with Eq. (17), we obtain the correction factor as

$$
g\left(\boldsymbol{r}_{0}, \boldsymbol{r}\right)=\frac{|J|}{2 \pi^{\frac{5}{2}} k^{2}}=\frac{4}{\pi^{\frac{5}{2}}}|\sin \beta|\left|\alpha_{u} \beta_{v}-\alpha_{v} \beta_{u}\right| .
$$

Using the focusing factor defined in Eq. (8) along with the derived correction factor of Eq. (21), we can reduce the integral transformation given in Eq. (7) to Eq. (6); thus, 


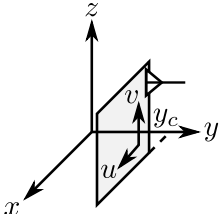

(a)

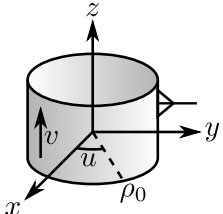

(b)

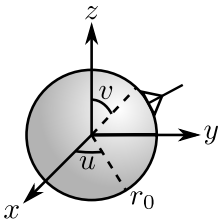

(c)
Fig. 3. Canonical antenna scanning surfaces. (a) Planar surface. (b) Cylindrical surface. (c) Spherical surface.

the desired image reconstruction is achieved. Eq. (21) is the general expression of the correction factor for an arbitrary scanning surface. For a parametric surface defined by an analytical formula, such as a plane, cylinder, or sphere, the exact expression for the partial derivative $\chi_{\xi}(u, v)$ can be obtained, and the exact correction factors for these surfaces can be derived, as discussed in Section II-D. However, for an arbitrary surface, obtaining the corresponding partial derivative $\chi_{\xi}(u, v)$ in analytical form may not be possible. In such a case, we must perform numerical computation of the partial derivative, as described in Section II-E.

The correction factor, $g\left(\boldsymbol{r}_{0}, \boldsymbol{r}\right)$, has a geometrical interpretation that it is related to the differential solid angle of the scanning surface with respect to the local coordinate system $\left(x^{\prime}, y^{\prime}, z^{\prime}\right)$. This differential solid angle represents the spatial sampling density on the scanning surface, and the correction factor functions as a regulator for image reconstruction such that the spatial sampling density on the surface is uniform. Note that the correction factor is not necessarily constant with respect to the surface parameters $(u, v)$ for a scanning surface that is sufficiently far from the spatial area to be imaged. This is because the irregularity of the spatial sampling density cannot be resolved by simply increasing the antenna distance from the imaged area; a simple example is the spherical scanning discussed in the numerical simulation in Section III.

\section{Exact Correction Factors for Special Surfaces}

In this section, we demonstrate how the derived general expression for the correction factor in Eq. (21) can be used to produce the specific correction factors for a planar, cylindrical, and spherical antenna scanning surface. Fig. 3 depicts the special surfaces considered in this study. In the following, we only provide the form of the partial derivatives $\alpha_{\xi}$ and $\beta_{\xi}$ ( $\xi \in\{u, v\}$ ) without substituting them into Eq. (21).

1) Planar Scanning: Fig. 3(a) shows a planar antenna scanning surface. We assume that the scanning plane is parallel to the $x$ - and $z$-axis, and fixed at $y=y_{c}$. The surface is defined by the following expression:

$$
x_{0}(u, v)=u, y_{0}(u, v)=y_{c}, z_{0}(u, v)=v .
$$

The partial derivatives $\alpha_{\xi}$ and $\beta_{\xi}$ are

$$
\begin{aligned}
& \alpha_{u}=-R_{y} / \rho^{2}, \alpha_{v}=0 \\
& \beta_{u}=R_{x} R_{y} /\left(R^{2} \rho\right), \beta_{v}=-\rho / R^{2}
\end{aligned}
$$

and the corresponding correction factor can be determined by Eq. (21).
2) Cylindrical Scanning: Fig. 3(b) shows a cylindrical antenna scanning surface expressed as

$$
x_{0}(u, v)=\rho_{0} \cos u, y_{0}(u, v)=\rho_{0} \sin u, z_{0}(u, v)=v,
$$

where $\rho_{0}$ denotes the constant radius of the cylinder. In this case, the partial derivatives $\alpha_{\xi}$ and $\beta_{\xi}$ are given as follows:

$$
\begin{aligned}
\alpha_{u} & =\frac{r_{0}}{\rho^{2}}\left(R_{x} \cos u+R_{y} \sin u\right), \alpha_{v}=0 \\
\beta_{u} & =\frac{R_{z} \rho_{0}}{R^{2} \rho}\left(R_{y} \cos u-R_{x} \sin u\right), \beta_{v}=-\frac{\rho}{R^{2}},
\end{aligned}
$$

and the corresponding correction factor can be computed by substituting these partial derivatives in Eq. (21). Note that the other relevant expressions for the cylindrical case have been derived in [10]-[13].

3) Spherical Scanning: Fig. 3(c) depicts a spherical antenna scanning surface expressed as

$$
\begin{aligned}
x_{0}(u, v) & =r_{0} \cos u \sin v, y_{0}(u, v)=r_{0} \sin u \sin v, \\
z_{0}(u, v) & =r_{0} \cos v
\end{aligned}
$$

where $r_{0}$ denotes the constant radius of the sphere. In this case, the partial derivatives $\alpha_{\xi}$ and $\beta_{\xi}$ are given by:

$\alpha_{u}=\frac{r_{0} \sin v}{\rho^{2}}\left(R_{x} \cos u+R_{y} \sin u\right)$

$\alpha_{v}=\frac{r_{0} \cos v}{\rho^{2}}\left(R_{x} \sin u-R_{y} \cos u\right)$

$\beta_{u}=\frac{R_{z} r_{0} \sin v}{\rho R^{2}}\left(R_{y} \cos u-R_{x} \sin u\right)$

$\beta_{v}=\frac{r_{0}}{\rho R^{2}}\left[R_{z} \cos v\left(R_{x} \cos u+R_{y} \sin u\right)+\rho^{2} \sin v\right]$,

and the correction factor can be calculated by applying Eq. (21).

\section{E. Numerical Correction Factor}

In this section, we present the proposed numerical computation of the correction factor, which can be applied for antenna scanning over an arbitrary surface. We considered a discrete subpatch of the scanning surface, as shown in Fig. 4. A discrete point on the subpatch is denoted by $\left(u_{m}, v_{n}\right)$, where $u_{m}, m \in\{1,2, \ldots, M\}$ and $v_{n}, n \in\{1,2, \ldots, N\}$ are the discrete spatial coordinates along the surface corresponding to $u$ and $v$, respectively. For notational simplicity, we define the indices $m=1$ and $n=1$ to represent the smallest spatial variables within this subpatch and $m=M$ and $n=N$ as the indices that represent the largest spatial variables within the subpatch. The spatial variables $\chi\left(u_{m}, v_{n}\right), \chi \in\left\{x_{0}, y_{0}, z_{0}\right\}$ are abbreviated as $\chi_{m, n}$. Using the aforementioned notation, we describe the following two types of numerical differentiation of the subpatch: the finite difference approximation and polynomial approximation.

1) Finite Difference Approximation: A simple solution for computing the Jacobian of Eq. (18) is to approximate the partial derivative with respect to the spatial variable using the finite difference. By employing the central difference, we can numerically compute the partial differences as

$$
\chi_{u}\left(u_{m}, v_{n}\right) \approx \frac{\chi_{m+1, n}-\chi_{m-1, n}}{u_{m+1}-u_{m-1}}
$$




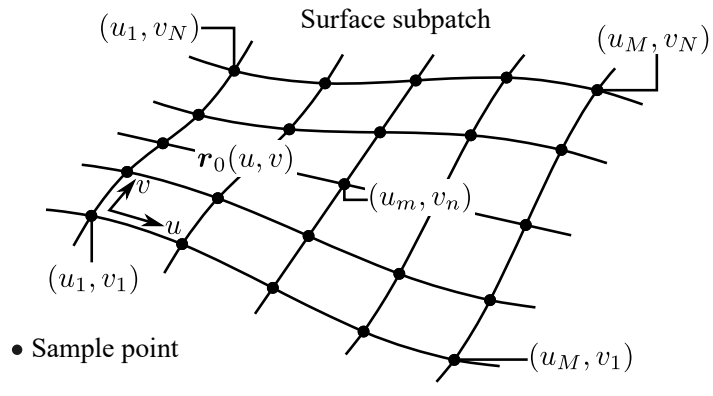

Fig. 4. Discrete subpatch of the scanning surface.

$$
\chi_{v}\left(u_{m}, v_{n}\right) \approx \frac{\chi_{m, n+1}-\chi_{m, n-1}}{v_{n+1}-v_{n-1}} .
$$

Using Eq. (28) and Eq. (19), the correction factor defined in Eq. (21) can be approximated and computed for an arbitrary scanning surface. Note that if the forward sample (i.e., $m+1$ and/or $n+1$ ) or backward sample (i.e., $m-1$ and/or $n-1$ ) is unavailable, the backward or forward differences, respectively, can be used instead of the central difference of Eq. (28).

2) Polynomial Approximation: As an alternative approach, polynomial-based numerical differentiation can be used to compute the correction factor. We considered the following polynomial expansion of $\chi_{m, n}$ at coordinates $\left(u_{m}, v_{n}\right)$.

$$
\chi_{m, n}=\sum_{p=0}^{K} \sum_{q=0}^{L} a_{p, q}^{(\chi)} u_{m}^{p} v_{n}^{q},
$$

where $a_{p, q}^{(\chi)}$ is the unknown expansion coefficient to be determined, $[\cdot]^{(\chi)}$ indicates that this coefficient is related to element $\chi \in\left\{x_{0}, y_{0}, z_{0}\right\}$ of the position vector $\boldsymbol{r}_{0}$, and $K$ and $L$ represent the order of the polynomial for $u_{m}$ and $v_{n}$, respectively.

By formulating Eq. (29) for all the sample points within the subpatch, the simultaneous equations with the unknown coefficients $a_{p, q}^{(\chi)}$ are obtained. Subsequently, by solving this equation for $a_{p, q}^{(\chi)}$, the antenna scanning surface can be approximated by polynomial equations within the subpatch, making it possible to evaluate the partial derivative $\chi_{\xi}$. In the following discussion, we provide a least-squares solution to Eq. (29).

Eq. (29) can be equivalently expressed by the following vector and matrix notation.

$$
\begin{aligned}
\chi_{m, n} & =\boldsymbol{u}_{m}^{T} \boldsymbol{A}^{(\chi)} \boldsymbol{v}_{n} \\
\boldsymbol{u}_{m} & =\left[1, u_{m}, u_{m}^{2}, \ldots, u_{m}^{p}, \ldots, u_{m}^{K}\right]^{T} \\
\boldsymbol{v}_{n} & =\left[1, v_{n}, v_{n}^{2}, \ldots, v_{n}^{q}, \ldots, v_{n}^{L}\right]^{T} \\
\boldsymbol{A}^{(\chi)} & =\left[\boldsymbol{a}_{0}^{(\chi)}, \boldsymbol{a}_{1}^{(\chi)}, \ldots \boldsymbol{a}_{p}^{(\chi)}, \ldots \boldsymbol{a}_{K}^{(\chi)}\right]^{T} \\
\boldsymbol{a}_{p}^{(\chi)} & =\left[a_{p, 0}^{(\chi)}, a_{p, 1}^{(\chi)}, \ldots, a_{p, q}^{(\chi)}, \ldots, a_{p, L}^{(\chi)}\right]^{T},
\end{aligned}
$$

where $[\cdot]^{T}$ represents the transpose operation. Eq. (30) can be reformulated into the following form.

$$
\begin{aligned}
\chi_{m, n} & =\left(\boldsymbol{u}_{m} \otimes \boldsymbol{v}_{n}\right)^{T}\left[\boldsymbol{a}_{0}^{(\chi) T}, \boldsymbol{a}_{1}^{(\chi) T}, \ldots \boldsymbol{a}_{p}^{(\chi) T}, \ldots \boldsymbol{a}_{K}^{(\chi) T}\right]^{T} \\
& =\boldsymbol{w}_{m, n}^{T} \boldsymbol{a}^{(\chi)}
\end{aligned}
$$

$$
\boldsymbol{a}^{(\chi)}=\left[\boldsymbol{a}_{0}^{(\chi) T}, \boldsymbol{a}_{1}^{(\chi) T}, \ldots \boldsymbol{a}_{p}^{(\chi) T}, \ldots \boldsymbol{a}_{K}^{(\chi) T}\right]^{T}
$$

where $\otimes$ denotes the Kronecker product. Considering the representation in Eq. (31) for all sample points within the subpatch, the following expression is obtained:

$$
\begin{aligned}
\boldsymbol{\chi} & =\boldsymbol{W} \boldsymbol{a}^{(\chi)} \\
\chi & =\left[\boldsymbol{\chi}_{1}^{T}, \boldsymbol{\chi}_{2}^{T}, \ldots, \boldsymbol{\chi}_{m}^{T}, \ldots, \boldsymbol{\chi}_{M}^{T}\right]^{T} \\
\boldsymbol{\chi}_{m} & =\left[\chi_{m, 1}, \chi_{m, 2}, \ldots, \chi_{m, n}, \ldots, \chi_{m, N}\right]^{T} \\
\boldsymbol{W} & =\left[\boldsymbol{W}_{1}^{T}, \boldsymbol{W}_{2}^{T}, \ldots \boldsymbol{W}_{m}^{T}, \ldots \boldsymbol{W}_{M}^{T}\right]^{T} \\
\boldsymbol{W}_{m} & =\left[\boldsymbol{w}_{m, 1}, \boldsymbol{w}_{m, 2}, \ldots, \boldsymbol{w}_{m, n}, \ldots, \boldsymbol{w}_{m, N}\right]^{T}
\end{aligned}
$$

The least-squares solution to the coefficient vector $\boldsymbol{a}^{(\chi)}$ can then be obtained as follows:

$$
\boldsymbol{a}^{(\chi)}=\left(\boldsymbol{W}^{T} \boldsymbol{W}\right)^{-1} \boldsymbol{W}^{T} \chi
$$

Using the coefficient vector $\boldsymbol{a}^{(\chi)}$, we can approximate the subpatch as

$$
\begin{aligned}
\chi(u, v) & \approx \boldsymbol{u}(u) \boldsymbol{A}^{(\chi)} \boldsymbol{v}^{T}(v) \\
\boldsymbol{u}(u) & =\left[1, u, u^{2}, \ldots, u^{p}, \ldots, u^{K}\right]^{T} \\
\boldsymbol{v}(v) & =\left[1, v, v^{2}, \ldots, v^{q}, \ldots, v^{L}\right]^{T} .
\end{aligned}
$$

Therefore, the partial difference $\chi_{\xi}(u, v)$ can be numerically evaluated as

$$
\begin{aligned}
\chi_{u}(u, v) & =\frac{\partial \chi(u, v)}{\partial u} \approx \boldsymbol{u}^{\prime}(u) \boldsymbol{A}^{(\chi)} \boldsymbol{v}^{T}(v) \\
\chi_{v}(u, v) & =\frac{\partial \chi(u, v)}{\partial v} \approx \boldsymbol{u}(u) \boldsymbol{A}^{(\chi)} \boldsymbol{v}^{\prime T}(v) \\
\boldsymbol{u}^{\prime}(u) & =\left[0,1,2 u, \ldots, p u^{(p-1)}, \ldots, K u^{(K-1)}\right]^{T} \\
\boldsymbol{v}^{\prime}(v) & =\left[0,1,2 v, \ldots, q v^{(q-1)}, \ldots, L v^{(L-1)}\right]^{T} .
\end{aligned}
$$

The aforementioned procedure is applied for $x_{0}, y_{0}$, and $z_{0}$ to obtain partial derivative $\chi_{\xi}(u, v)$. Subsequently, the correction factor of Eq. (21) and the focusing factor of Eq. (8) are numerically computed. A similar procedure was repeated for all the subpatches within the scanning surface. In the numerical simulation presented in Section III and the experimental validation presented in Section IV, we use a polynomial approximation to compute the numerical correction factor.

\section{F. Radar Cross-Section Computation}

The final step of image-based NFFFT is to compute the far-field RCS from the reconstructed radar image. We assume that the image is generated using Eq. (7) with the correction factor given by Eq. (21) and considered to be a sum of the ideal images defined by Eq. (6). Therefore, the scattered field at an arbitrary location $\boldsymbol{r}_{0}$ can be computed using Eq. (2) as follows:

$$
\begin{aligned}
E^{s}\left(k, \boldsymbol{r}_{0}\right)=\frac{k^{2}}{\sqrt{4 \pi}} \int_{-\infty}^{\infty} & \int_{-\infty}^{\infty} \int_{-\infty}^{\infty} \psi(x, y, z) \\
& \cdot \frac{e^{-2 j k\left|\boldsymbol{r}_{0}-\boldsymbol{r}\right|}}{\left|\boldsymbol{r}_{0}-\boldsymbol{r}\right|^{2}} d x d y d z
\end{aligned}
$$


Note that we have defined $P\left(k, \boldsymbol{r}_{0}, \boldsymbol{r}\right)=1$. Moreover, in the following, we assume that the location $\boldsymbol{r}_{0}=\left(x_{0}, y_{0}, z_{0}\right)$ is the spherical coordinate defined as follows:

$$
\begin{aligned}
& x_{0}\left(\phi_{0}, \theta_{0}\right)=r_{0} \cos \phi_{0} \sin \theta_{0}, y_{0}\left(\phi_{0}, \theta_{0}\right)=r_{0} \sin \phi_{0} \sin \theta_{0}, \\
& z_{0}\left(\phi_{0}, \theta_{0}\right)=r_{0} \cos \theta_{0},
\end{aligned}
$$

where $r_{0}, \phi_{0}$, and $\theta_{0}$ are the radius, the azimuth angle, and the zenith angle, respectively.

If the observation point $\boldsymbol{r}_{0}$ lies in the far-field region, that is, $\boldsymbol{r}_{0} \gg|\boldsymbol{r}|$, the distance between points $\boldsymbol{r}$ and $\boldsymbol{r}_{0}$ can be approximated as

$$
\left|\boldsymbol{r}_{0}-\boldsymbol{r}\right| \approx r_{0}-\widehat{\boldsymbol{r}}_{0} \cdot \boldsymbol{r}
$$

where $\widehat{\boldsymbol{r}}_{0}=\boldsymbol{r}_{0} / r_{0}$. Using Eq. (38), we obtain the following far-field approximation of the scattered field $E^{s}\left(k, \boldsymbol{r}_{0}\right)$ :

$$
\begin{aligned}
E^{s}\left(k, \boldsymbol{r}_{0}\right) \approx & \frac{k^{2} e^{-j 2 k r_{0}}}{r_{0}^{2} \sqrt{4 \pi}} \int_{-\infty}^{\infty} \int_{-\infty}^{\infty} \int_{-\infty}^{\infty} \psi(x, y, z) \\
& =\frac{k^{2} e^{-j 2 k r_{0}}}{r_{0}^{2} \sqrt{4 \pi}} \mathcal{F}_{(x, y, z)}^{-1}[\psi(x, y, z)],
\end{aligned}
$$

where $\boldsymbol{k}_{r}\left(k, \phi_{0}, \theta_{0}\right)=2 k \widehat{\boldsymbol{r}}_{0}$ represents the spatial frequency, and $\mathcal{F}_{(x, y, z)}^{-1}[\cdot]$ is the 3 -D inverse Fourier transform with respect to the spatial variables, $(x, y, z)$.

The far-field expression in Eq. (39) can be used to derive the RCS defined as follows:

$$
\sigma\left[\boldsymbol{k}_{r}\left(k, \phi_{0}, \theta_{0}\right)\right]=\lim _{r_{0} \rightarrow \infty} 4 \pi r_{0}^{2}\left|\frac{E^{s}\left(k, \boldsymbol{r}_{0}\right)}{E_{0}^{i}\left(k, \boldsymbol{r}_{0}\right)}\right|^{2},
$$

where $E_{0}^{i}\left(k, \boldsymbol{r}_{0}\right)$ represents the incident field given by

$$
E_{0}^{i}\left(k, \boldsymbol{r}_{0}\right)=\frac{e^{-j k r_{0}}}{r_{0}} .
$$

Substituting Eq. (39) and Eq. (41) into Eq. (40), we obtain the following representation of the RCS in terms of the 3-D spatial image $\psi(x, y, z)$ :

$$
\sigma\left[\boldsymbol{k}_{r}\left(k, \phi_{0}, \theta_{0}\right)\right]=k^{4}\left|\mathcal{F}_{(x, y, z)}^{-1}[\psi(x, y, z)]\right|^{2}
$$

Therefore, once the 3-D radar image is reconstructed, the far-field RCS is obtained by computing its inverse Fourier transform.

Note that we have assumed that the spatial image $\psi(x, y, z)$ is uniquely represented as the sum of the ideal image defined in Eq. (6), independent of the measurement parameters, such as the frequency; bandwidth; and shape and size of the scanning surface. However, in the actual measurement, the bandwidth is finite and the scanning surface may not completely enclose the area to be imaged. Consequently, the available RCS is limited within the spatial frequency $\left(\boldsymbol{k}_{r}\right)$ domain support, as can be seen from the simulation results with spherical scanning in Section III and the experimental result with cylindrical scanning in Section IV. Therefore, one should be aware of the available spatial frequency domain support when using the image-based NFFFT method.

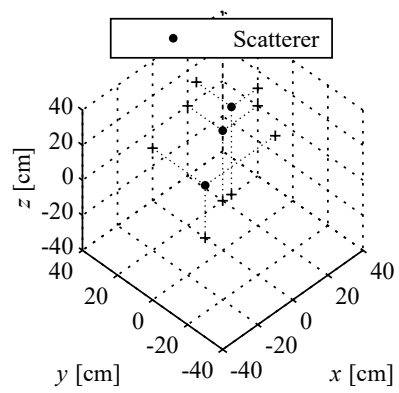

(a)

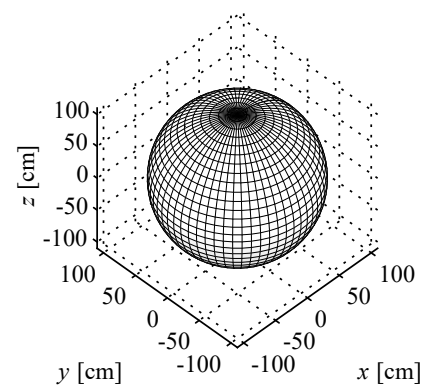

(b)
Fig. 5. Scatterer location and scanning surfaces. (a) Scatterer location. (b) spherical surface.

TABLE I

SIMULATION PARAMETERS.

\begin{tabular}{l|l}
\hline Center frequency & $10.2 \mathrm{GHz}$ \\
Bandwidth & $4 \mathrm{GHz}$ \\
Frequency step & $10 \mathrm{MHz}$ \\
Antenna scanning surface & Sphere \\
Angular interval in azimuth & $u \in\left[-180^{\circ}, 180^{\circ}\right]$ \\
Angular interval in zenith & $v \in\left[0^{\circ}, 180^{\circ}\right]$ \\
Angular step & $0.8^{\circ}$ \\
Radius of the surface & $r_{0}=1 \mathrm{~m}$ \\
Number of points in a subpatch & $M=N=11$ \\
Order of the polynomial & $K=L=2$ \\
Scatterer location & $\boldsymbol{r}_{1}=(0,10,-10) \mathrm{cm}$ \\
& $\boldsymbol{r}_{2}=(20,20,0) \mathrm{cm}$ \\
& $\boldsymbol{r}_{3}=(25,20,10) \mathrm{cm}$ \\
Radius of the spherical scatterer & $a_{1}=a_{2}=a_{3}=1 \mathrm{~mm}$ \\
Voxel spacing of the spatial image & $4 \mathrm{~mm}$ \\
\hline
\end{tabular}

\section{NUMERICAL SimUlation}

In this section, we demonstrate the proposed image-based NFFFT using the discrete scatterer model defined in Eq. (5). A spherical scanning surface was used in the simulation.

\section{A. Overview of the Simulation}

Fig. 5(a) and (b) show the location of the point scatterers and the spherical scanning surface, respectively, and Table I lists the simulation parameters. The three spherical scatterers having equivalent radius of $1 \mathrm{~mm}\left(k a_{i}<0.3\right)$ were placed at the different 3-D spatial locations. To generate the received signal for these scatterers, we used Eq. (4) and Eq. (5), with $i \in\{1,2,3\}$, and $\boldsymbol{r}_{i}$ defined in Table I. The correction factor was computed using the polynomial-based numerical differentiation described in Section II-E2. We tested both the exact correction factor and numerical correction factor for the spherical scanning surface and confirmed that similar results were obtained. The frequency parameters in Table I were selected to ensure consistency with the experimental validation given in Section IV. The antenna pattern was assumed to be isotropic, such that $P\left(k, \boldsymbol{r}_{0}, \boldsymbol{r}\right)=1$.

We compared the reconstructed RCS with the theoretical RCS of a cloud of point scatterers of reflectivity $C_{i}$, given as follows:

$$
\sigma=k^{4}\left|\sum_{i} C_{i} \exp \left(j k_{x} x_{i}+j k_{y} y+j k_{z} z\right)\right|^{2} .
$$


The spatial frequencies $\left(k_{x}, k_{y}, k_{z}\right)$ were determined using Eq. (16) with $\alpha \rightarrow \phi_{0}$ and $\beta \rightarrow \theta_{0}$. To quantify the prediction error of the NFFFT algorithm, we defined the average difference between the theoretical far-field RCS and predicted RCS on a decibel scale as follows:

$$
\widehat{\sigma}_{\zeta}^{\mathrm{dB}}=\frac{1}{N_{\zeta}} \sum_{n=1}^{N_{\zeta}}\left|\sigma_{\zeta[n]}^{\mathrm{dB}}-\widetilde{\sigma}_{\zeta[n]}^{\mathrm{dB}}\right|
$$

where $\sigma_{\zeta[n]}^{\mathrm{dB}}$ and $\widetilde{\sigma}_{\zeta[n]}^{\mathrm{dB}}\left(\zeta \in\left\{\phi_{0}, \theta_{0}\right\}\right)$ are the $n$th samples of the true and predicted RCS patterns in the decibel scale, respectively, in the azimuth $\left(\zeta=\phi_{0}\right)$ or zenith $\left(\zeta=\theta_{0}\right)$ direction; $N_{\zeta}$ is the number of samples in each direction.

\section{B. Simulation Results and Discussion}

Figs. 6 and 7 show the simulation results with and without $\left(g\left(\boldsymbol{r}_{0}, \boldsymbol{r}\right)=1\right)$ the correction factor, respectively. In these figures, (a), (b), (c), and (d) depict the reconstructed 3D spatial image, spatial-frequency spectrum of the spatial image, azimuth RCS pattern at the zenith angle, $\theta_{0}=90^{\circ}$, and zenith RCS pattern at the azimuth angle, $\phi_{0}=0^{\circ}$, respectively. The spatial images and spatial frequency spectra were normalized using the maximum value of each image. The spatial frequency spectra within the $\left(k_{x}, k_{y}\right)$-plane and $\left(k_{x}, k_{z}\right)$-plane are shown in these figures. All the RCS patterns were computed for $10 \mathrm{GHz}$. The solid lines represent the farfield RCS reconstructed using the NFFFT algorithm, and the dashed lines represent the theoretical RCS determined from Eq. (43).

Note that in this spherical scanning case, we can obtain the RCS at an arbitrary combination of the azimuth and zenith angle because the scanning surface completely enclosed the spatial area in which the scatterers were located. However, because the frequency bandwidth is finite, RCS is only available within this bandwidth, as mentioned in Section II-F. This corresponds to the spatial frequency spectra shown in Figs. 6(b) and 7(b) having annulus-like supports in $\left(k_{x}, k_{y}\right)$ - and $\left(k_{x}, k_{z}\right)$-plane, where the widths of the circular bands are dictated by the frequency bandwidth.

As shown in Fig. 6(a), without using the correction factor, the undesired artifact appeared around the scatterers. The spatial image of a point target is expected to be symmetric around its location; however, the reconstructed image was blurred and asymmetric owing to the artifact. We can see from Fig. 6(b) that the spatial frequency spectrum is concentrated around zenith angles $\theta_{0}=0^{\circ}$ and $\theta_{0}=180^{\circ}$ in $\left(k_{x}, k_{z}\right)$-plane. This is because although angular sample spacing is uniform in both the azimuth and zenith directions, the spatial density of the sampling points on the spherical surface becomes higher around these zenith angles. Owing to this effect, a significant error was observed in the reconstructed zenith RCS, as shown in Fig. 6(d). As discussed in Section II-C, this error cannot be circumvented by simply making the radius of the scanning sphere large; the introduction of the correction factor is essential to compensate for the irregularity of the spatial sampling density on the sphere.

As shown in Fig. 7(a), an obvious improvement was obtained by using the proposed correction factor; each point

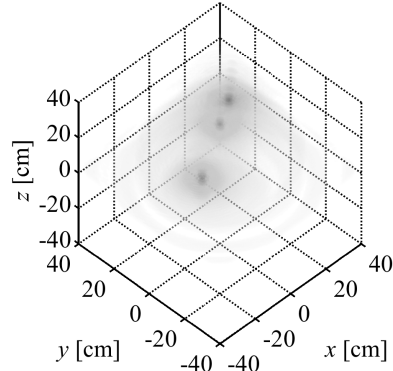

(a)

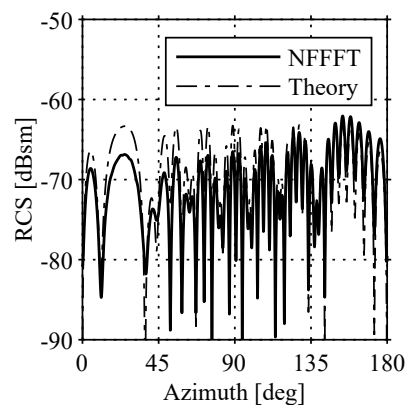

(c)

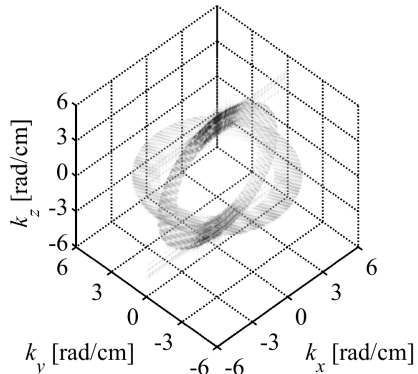

(b)

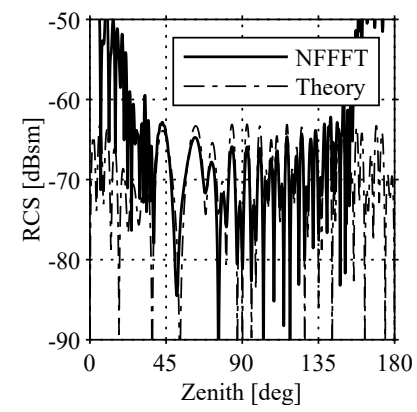

(d)
Fig. 6. NFFFT with a spherical scanning surface (conventional, simulation). (a) Spatial image. (b) Spatial frequency spectrum. (c) Azimuth RCS. (d) Zenith RCS.

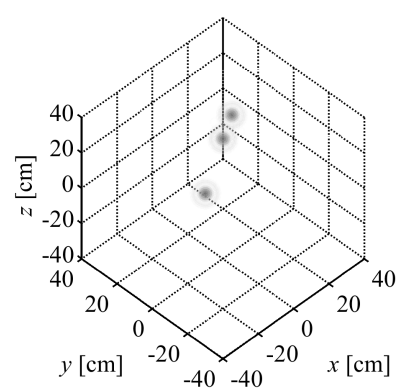

(a)

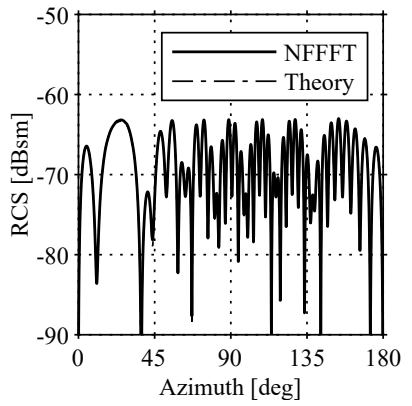

(c)

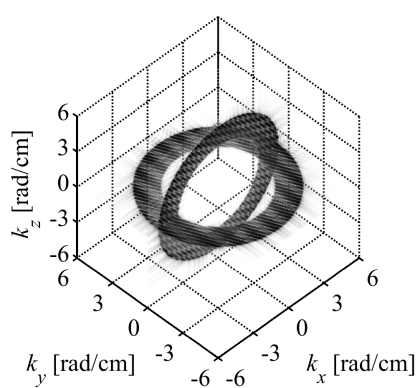

(b)

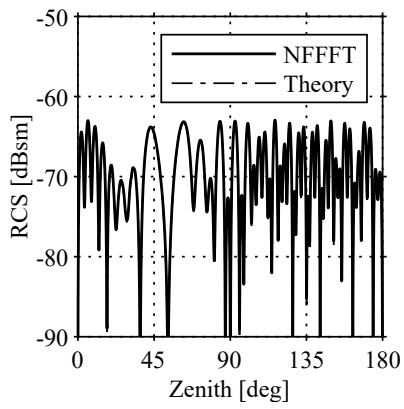

(d)
Fig. 7. NFFFT with a spherical scanning surface (improved, simulation). (a) Spatial image. (b) Spatial frequency spectrum. (c) Azimuth RCS. (d) Zenith RCS.

target showed asymmetric response with respect to the corresponding scatterer location. Consequently, the spatial frequency spectrum shown in Fig. 7(b) is uniformly distributed over all the azimuth and zenith angles. The resultant RCS values showed excellent agreement with the theoretical values, as shown in Fig. 7(c) and (d). This improvement can be confirmed form the averaged RCS prediction error defined as Eq. (44). Comparing the NFFFT results with and without the 


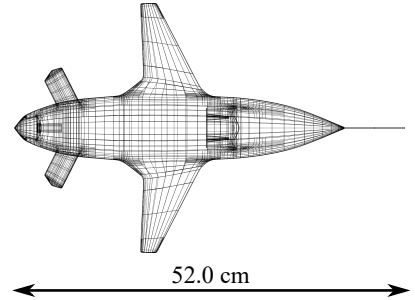

(a)

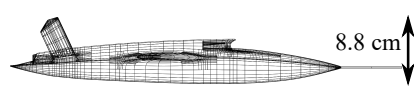

(c)

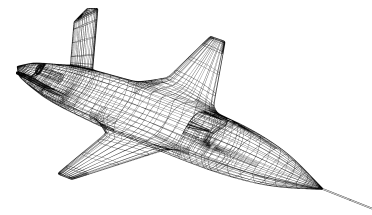

(b)

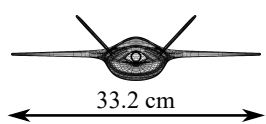

(d)
Fig. 8. Aircraft model used in the experiment. (a) Top view. (b) Perspective view. (c) Side view. (d) Front view.

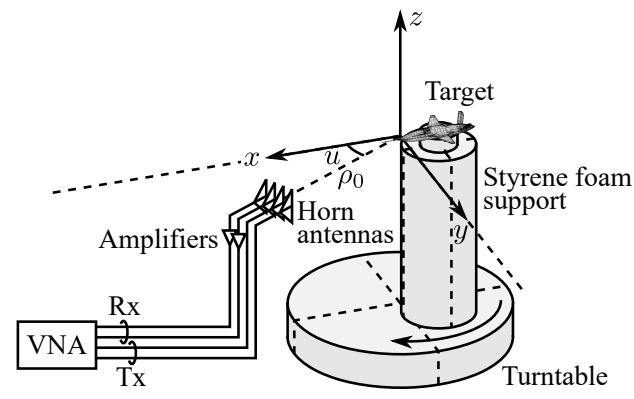

Fig. 9. Geometry of the experiment. The target is intentionally off-centered to demonstrate the effectiveness of the proposed correction factor.

correction factor, the prediction error was reduced from $2.9 \mathrm{~dB}$ to $0.1 \mathrm{~dB}$ for the azimuth RCS and from $8.8 \mathrm{~dB}$ to $0.2 \mathrm{~dB}$ for the zenith RCS.

\section{EXPERIMENTAL VALIDATION}

In this section, we discuss the experimental validation of the proposed algorithm. The experiment based on the cylindrical scanning was conducted in an anechoic chamber, and a metallic aircraft model was used for the measurement.

\section{A. Overview of the Experiment}

Fig. 8 illustrates the geometry of the aircraft model used in this experiment, where (a), (b), (c), and (d) show the top, perspective, side, and front view, respectively. Figs. 9 and 10 show the setup and photographs of the measurement system, respectively. The target was fixed on a styrene foam support, and the support was placed on a turntable for azimuth rotation. As shown in Fig. 9, the target was intentionally off-centered to demonstrate the effectiveness of the proposed correction factor, where the center of the model was $(-15,15,0) \mathrm{cm}$, and the front of the aircraft was directed toward the $x$-axis. Thus, the target was strongly asymmetric about the origin of the coordinate system.

Four standard gain horn antennas (Narda Model 640) were used as transmitting and receiving antennas. The two horn antennas were horizontally polarized, whereas the other antennas were vertically polarized. These antennas were mounted

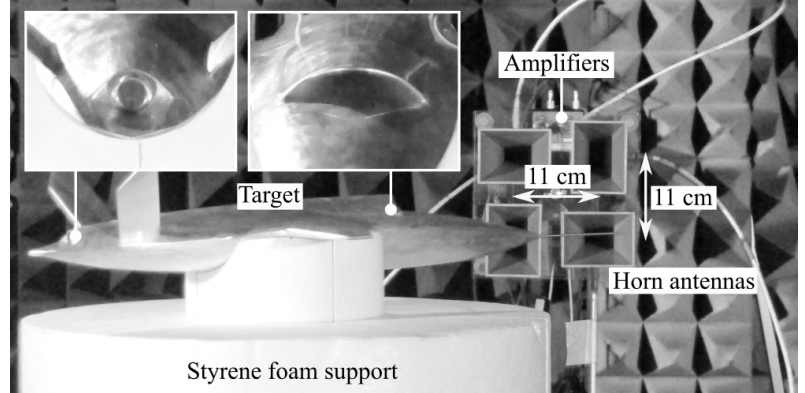

Fig. 10. Photographs of the experimental setup. The target was placed on a styrene foam support. Four horn antennas were used for transmission and reception.

on a vertical scanner that could move the antennas in the vertical (height) direction. We performed cylindrical antenna scanning using a turntable and vertical scanner. A vector network analyzer (VNA, Keysight N5242A) was used as the transmitter and receiver. Under this experimental configuration, two co-polarized channels, namely, the HH- (horizontally polarized transmission and reception) and VV-channels (vertically polarized transmission and reception), were measured. We assumed that the monostatic approximation was valid, where the monostatic antenna was assumed to be located at the center of the antenna separation.

Table II lists the experimental parameters. The frequency parameters similar to the previous numerical simulation were employed for this experiment. To compensate for the effect of the antenna pattern, the theoretical pattern of the horn antenna was computed using an analytical expression given in [17]. Because the cylindrical scanning surface was at the far-field distance with respect to the vertical size of the aircraft model, the cylindrical scanning was not essential to measure the azimuth RCS. Therefore, the cylindrical scanning was just for obtaining the zenith RCS and for demonstrating the concept of RCS determination from the 3-D synthetic aperture imaging.

Because we were unable to measure the far-field RCS directly owing to the spatial limitation of the anechoic chamber used for this measurement, the far-field RCS of the target was computed using a numerical electromagnetic solver using the multilevel fast multipole method (MLFMM). We assumed the MLFMM results to be the true far-field RCS of the experimental model to evaluate the average RCS-prediction error defined in Eq. (44).

\section{B. Experimental Results and Discussion}

Fig. 11(a) and (b) show the HH-polarized 3-D spatial image generated using the proposed correction factor and the corresponding spatial frequency spectrum, respectively. The spatial image and spatial frequency spectrum were normalized by the maximum value of each image. As shown in Fig. 11(a), backscattering from the aircraft model was clearly imaged. In Fig. 11(b), the spatial frequency spectra within the $\left(k_{x}, k_{y}\right)$-plane and the $\left(k_{x}, k_{z}\right)$-plane are displayed. Although the spatial frequency spectrum shown in Fig. 11(b) has an annulus-like shape in $\left(k_{x}, k_{y}\right)$-plane, the spectrum appears 
TABLE II

EXPERIMENTAL PARAMETERS.

\begin{tabular}{l|l}
\hline Center frequency & $10.2 \mathrm{GHz}$ \\
Bandwidth & $4 \mathrm{GHz}$ \\
Frequency step & $10 \mathrm{MHz}$ \\
Polarization & $\mathrm{HH}, \mathrm{VV}$ \\
Antenna scanning surface & $\mathrm{Cylinder}$ \\
Angular interval in azimuth & $u \in\left[-180^{\circ}, 180^{\circ}\right]$ \\
Angular step & $0.8^{\circ}$ \\
Height ( $z$-direction) interval & $v \in[-25,25] \mathrm{cm}$ \\
Height ( $z$-direction) step & $1 \mathrm{~cm}$ \\
Radius of the surface & $\rho_{0}=1 \mathrm{~m}$ \\
Number of points in a subpatch & $M=N=11$ \\
Order of the polynomial & $K=L=2$ \\
Target & Aircraft model \\
Voxel spacing of the spatial image & $4 \mathrm{~mm}$ \\
\hline
\end{tabular}

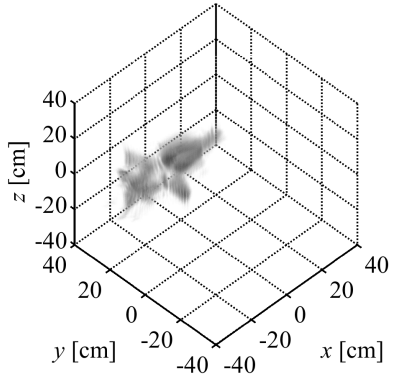

(a)

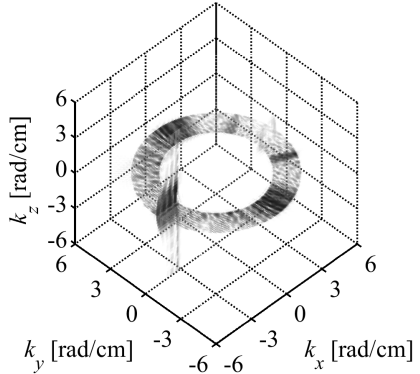

(b)
Fig. 11. Spatial image and spatial frequency spectrum (experiment, HHpolarization, improved). (a) Spatial image. (b) Spatial frequency spectrum.

to be limited within a certain range in $k_{z}$-domain. This is because unlike the spherical case in Section III, a cylinder with a finite length cannot completely enclose the target under test. Therefore, RCS is only available within the range of the zenith angle dictated by the $k_{z}$-domain support, as discussed in Section II-F.

Figs. 12 and 13 show the azimuth RCS for $\mathrm{HH}$ and VV polarization, respectively, where (a) and (b) represent the conventional and improved NFFFT, respectively. All of these RCS patterns were computed for $10 \mathrm{GHz}$. The solid lines represent the far-field RCS reconstructed using the NFFFT algorithm, and the dashed lines represent the far-field RCS computed by the MLFMM solver. Thus, the improvement of the prediction accuracy of the azimuth RCS by using the proposed correction factor for both polarization channels can be confirmed. Comparing Figs. 12 and 13, it can be seen that the HH-polarized channel shows much weaker backscattering around the azimuth angle $\phi_{0}=0^{\circ}$ in contrast to the strong backscattering around that angle in the VV-polarized channel. This can be attributed to the geometry of the aircraft model, where a horizontal aperture (air intake) was present in front of the model (see Fig. 10).

Figs. 14 and 15 show similar figures for zenith RCS for $\mathrm{HH}$ and VV polarization, respectively. Both Figs. 14 and 15 show the improvement in prediction accuracy of the zenith RCS by using the proposed correction factor although the difference between the conventional and the improved NFFFT is relatively unclear compared to those in the azimuth RCS.

Fig. 16(a) and (b) show the averaged prediction error for

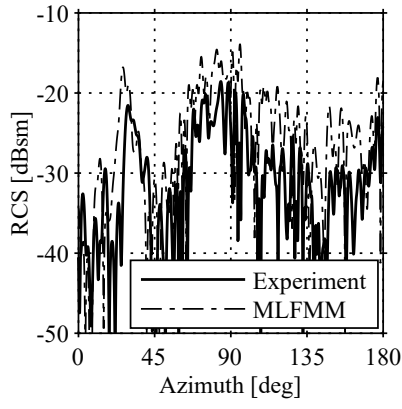

(a)

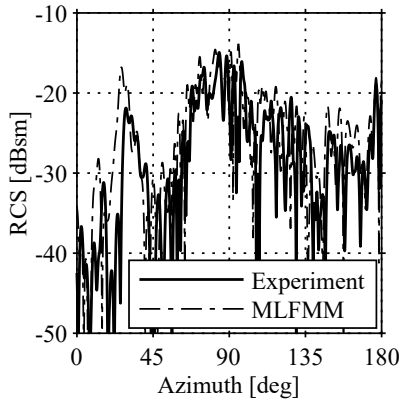

(b)
Fig. 12. Azimuth RCS of the aircraft model (experiment, HH-polarization). (a) Conventional. (b) Improved.

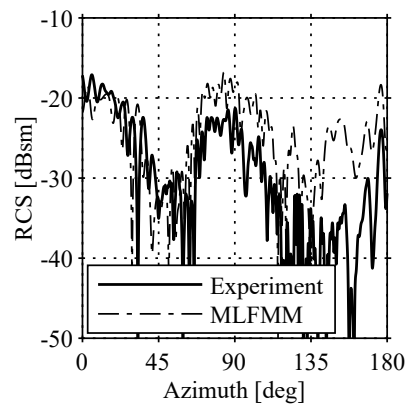

(a)

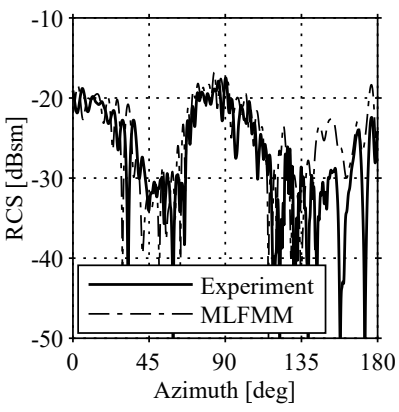

(b)
Fig. 13. Azimuth RCS of the aircraft model (experiment, VV-polarization). (a) Conventional. (b) Improved.

the azimuth and zenith RCS. We only considered the zenith angle within the interval $\theta_{0} \in\left[80^{\circ}, 100^{\circ}\right] \cup\left[-100^{\circ},-80^{\circ}\right]$ for the computation of Eq. (44) because the data outside these intervals were unavailable owing to the finite cylinder length. From Fig. 16, both the azimuth and the zenith RCS demonstrate the improvement by using the proposed correction factor for both the HH- and VV-polarization channels. From these results, we experimentally confirmed the effectiveness of the proposed correction factor.

\section{CONClusion}

In this study, we proposed an image-based NFFFT algorithm for far-field RCS determination that can accommodate an arbitrary antenna scanning surface. We derived a generalized expression of the correction factor and focusing factor for image reconstruction. We proposed the use of numerical differentiation to compute the correction factor for an arbitrary scanning surface. The numerical simulation and experimental results confirmed the validity of the proposed algorithm. In future work, an extension of the proposed approach to bistatic cases and efficient numerical implementation of the imaging algorithm should be considered.

\section{REFERENCES}

[1] D. G. Falconer, "Extrapolation of near-field RCS measurements to the far zone," IEEE Trans. Antennas Propag., vol. 36, no. 6, pp. 822-829, Jun. 1988. 


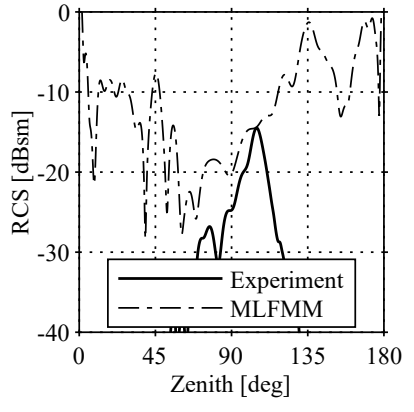

(a)

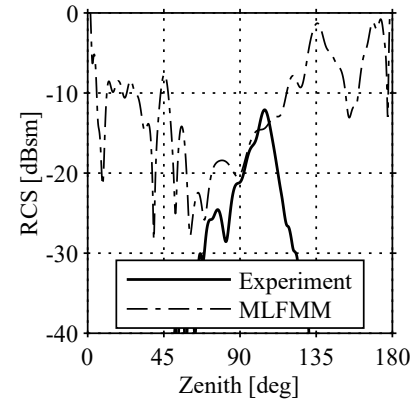

(b)
Fig. 14. Zenith RCS of the aircraft model (experiment, HH-polarization). (a) Conventional. (b) Improved.

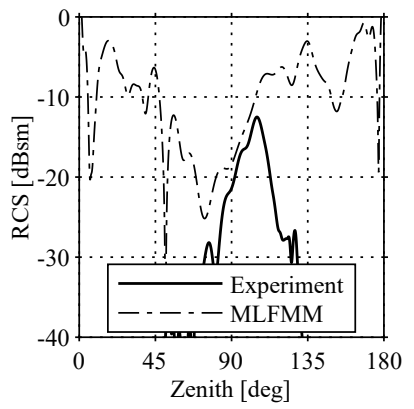

(a)

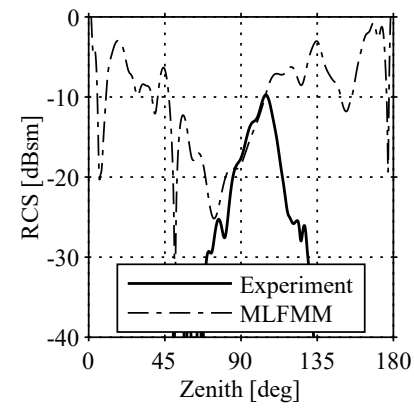

(b)
Fig. 15. Zenith RCS of the aircraft model (experiment, VV-polarization). (a) Conventional. (b) Improved.

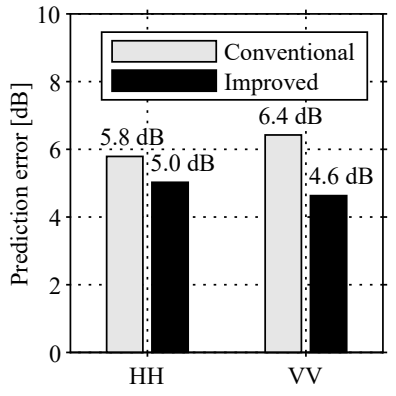

(a)

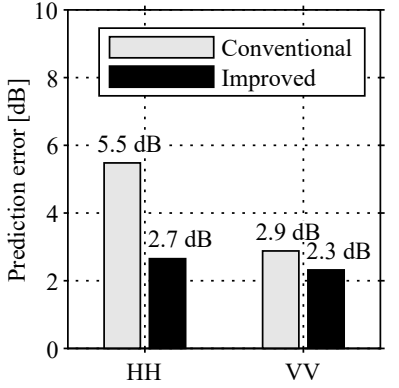

(b)
Fig. 16. Averaged RCS prediction error (experiment). (a) Azimuth RCS. (b) Zenith RCS.

[2] G. Schnattinger, R. A. M. Mauermayer, and T. F. Eibert, "Monostatic Radar Cross Section Near-Field Far-Field Transformations by Multilevel Plane-Wave Decomposition," IEEE Trans. Antennas Propag., vol. 62, no. 8, pp. 4259-4268, Aug. 2014.

[3] O. Neitz, R. A. M. Mauermayer, and T. F. Eibert, "3-D Monostatic RCS Determination From Multistatic Near-Field Measurements by PlaneWave Field Synthesis," IEEE Trans. Antennas Propag., vol. 67, no. 5, pp. 3387-3396, May 2019.

[4] S. Omi, M. Hirose, M. Ameya, and S. Kurokawa, "Plane-Wave Synthesis Employing Propagating Plane-Wave Expansion for 3-D and 2-D RCS Prediction Including the Multiple Scattering Effects," IEEE Trans. Antennas Propag., vol. 69, no. 8, pp. 4827-4835, Aug. 2021.

[5] J. W. Odendaal and J. Joubert, "Radar cross section measurements using near-field radar imaging," IEEE Trans. Instrum. Meas., vol. 45, no. 6, pp. 948-954, Dec. 1996.

[6] A. Broquetas, J. Palau, L. Jofre, and A. Cardama, "Spherical wave near-field imaging and radar cross-section measurement," IEEE Trans. Antennas Propag., vol. 46, no. 5, pp. 730-735, May 1998.

[7] T. Vaupel and T .F. Eibert, "Comparison and application of near-field
ISAR imaging techniques for far-field radar cross section determination," IEEE Trans. Antennas Propag., vol. 54, no. 1, pp. 144-151, Jan. 2006.

[8] T. Vaupel and F. Weinmann, "Validation of a 3-D near-field ISAR imaging technique with far-field RCS extraction by means of a hybrid GO-PO/PTD ray tracing algorithm," in 2009 3rd European Conference on Antennas and Propagation, Mar. 2009, pp. 691-695.

[9] A. V. Osipov, H. Kobayashi, and H. Suzuki, "An Improved Image-Based Circular Near-Field-to-Far-Field Transformation," IEEE Trans. Antennas Propag., vol. 61, no. 2, pp. 989-993, Feb. 2013.

[10] A. V. Osipov, H. Kobayashi and H. Suzuki, "An Improved ImageBased Near-Field-to-Far-Field Transformation for Cylindrical Scanning Surfaces," in Proceedings of 30th General Assembly and Scientific Symposium of International Union of Radio Science (URSI), Istanbul, Turkey, Aug. 13-20, 2011, pp. 1-4.

[11] A. V. Osipov, H. Kobayashi and H. Suzuki, "An Improved Cylindrical NFFFT for Compact Measurement Facilities." in Proceedings of AsiaPacific Microwave Conference (APMC 2012), Kaohsiung, Taiwan, Dec. 4-7, 2012, pp. 1304-1306.

[12] T. Vaupel, "An improved 3-D near-field ISAR imaging technique with extended far-field RCS extraction," in 2015 IEEE International Symposium on Antennas and Propagation USNC/URSI National Radio Science Meeting, Jul. 2015, pp. 699-700

[13] T. Vaupel, "A Fast 3-D Cylindrical Scanning Near-Field ISAR Imaging Approach With Extended Far-Field RCS Extraction Based on a Modified Focusing Operator," in Proceedings of EUSAR 2016: 11th European Conference on Synthetic Aperture Radar, Jun. 2016, pp. 97-101.

[14] I. J. LaHaie, "Overview of an image-based technique for predicting farfield radar cross section from near-field measurements," IEEE Antennas and Propagation Magazine, vol. 45, no. 6, pp. 159-169, Dec. 2003.

[15] I. J., LaHaie, "An improved version of the circular near field-to-far-field transformation (CNFFFT)," Proceedings of the 27th Annual Meeting of the Antenna Measurement Techniques Association (AMTA'05), pp. 196201, Newport, RI, 2005.

[16] G. T. Ruck, D. E. Barrick, W. D. Stuart, and C. K. Krichbaum, Radar Cross Section Handbook, Vol. 1, Plenum Press, New York, 1970.

[17] C. A. Balanis, Antenna Theory: Analysis and Design, 4th edition, Wiley, 2016.

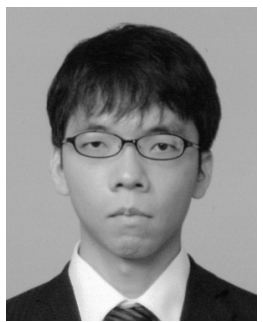

Takuma Watanabe (Member, IEEE) received the B.E., M.E., and Ph.D. degrees in electrical and information engineering from Niigata University, Niigata, Japan, in 2010, 2012, and 2015, respectively.

From 2015 to 2017, he worked with the Central Research Institute of Electric Power Industry, Yokosuka, Japan. From 2017 to 2018, he worked with PASCO Corporation (Tokyo, Japan). Since 2018, he has been with Fujitsu Ltd., Kawasaki, Japan, and since 2021, he has been with Fujitsu System Integration Laboratories Ltd., Kawasaki, Japan, where he is a research scientist. His research interests include image reconstruction problems, synthetic aperture radar (SAR) signal processing, array signal processing, and electromagnetic scattering problems.

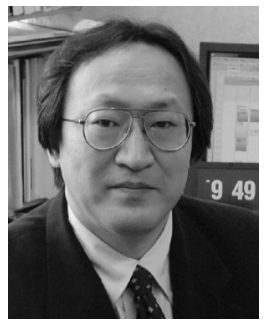

Hiroyoshi Yamada (Member, IEEE) received the B.E., M.E., and Ph.D. degrees in electronic engineering from Hokkaido University, Sapporo, Japan, in 1988, 1990, and 1993, respectively.

Since 1993, he has been a member of the Faculty of Engineering at Niigata University, Niigata, Japan, where he is currently a professor. From 2000 to 2001, he was a visiting scientist at the Jet Propulsion Laboratory, California Institute of Technology, Pasadena, California, USA. His research interests include array signal processing, polarimetric radar interferometry, and high-resolution techniques. 